\title{
Problematización acerca de la articulación entre extensión, docencia e investigación en la UNLP y su repercusión en la formación universitaria.
}

Problematization about articulation between community programs, teaching activities and research at University of La Plata and its impact on higher university education.

Santiago Héctor Fusé*

https://orcid.org/0000-0003-3298-7383

prof.santiagofuse@gmail.com

Ingrid Denise Karpenko Wilman***

https://orcid.org/0000-0001-5933-6738

karpenkoingrid5@gmail.com
María Florencia Henning**

https://orcid.org/0000-0002-9487-7748

florhenningedu@gmail.com

Magalí Pellon Maison****

https://orcid.org/0000-0002-7981-5258

magalipellon@med.unlp.edu.ar

Universidad Nacional de La Plata | Argentina

\section{RESUMEN}

Este trabajo se focaliza en la problematización acerca de la articulación entre las distintas funciones de la Universidad y fue realizado en el contexto de la acreditación del seminario "Políticas de articulación, docencia, investigación, extensión, y transferencia", de la Especialización en Docencia Universitaria de la Universidad Nacional de La Plata. Mediante el debate y la discusión grupal hemos identificado distintas problemáticas, que dificultan la formación integral de los y las estudiantes, como la desinformación de la planta docente en cuanto a la estructuración y gestión de la extensión universitaria, la falta de reconocimiento de la actividad extensionista y su escasa incorporación en los currículos de las carreras, la formación profesional descontextualizada, el uso no democrático de los espacios de trabajo en las distintas unidades académicas o las escasas iniciativas para promover el aprendizaje desde la práctica. Para cada uno de los problemas encontrados se proponen estrategias para su abordaje, con el propósito de pensar la formación y la educación como una construcción social del conocimiento. Concluimos que, resulta necesario promover el conocimiento a partir de la vinculación entre la extensión, la investigación y la docencia, tres funciones de la Universidad estrechamente vinculadas, pero no reconocidas todavía de igual modo.

\section{PALABRAS CLAVE} extensión universitaria, integralidad, articulación, docencia universitaria 
KEY WORDS

community programs, integrity, articulation, teaching activities

\section{ABSTRACT}

This work focuses on the problematization about the articulation between the different functions of the University, and was carried out in the context of the accreditation of the seminar "Policies of articulation, teaching, research, community programs and knowledge translation", of the "Specialization in teaching activities at the National University of La Plata". Through debate and group discussion, we have identified problems that hinder the comprehensive training of students, such as the misinformation of the teaching staff regarding the structuring and management of community programs, the lack of recognition of the community programs activity and its scarce incorporation into career curricula, decontextualized professional training, undemocratic use of work spaces in different academic units or few initiatives to promote learning from practice. For each of the problems found, strategies are proposed for their approach, with the purpose of thinking of training and education as social construction of knowledge. We conclude that it is necessary to promote knowledge from the link between community programs, research and teaching activities, three roles of the University closely linked, but not yet recognized in the same way. 


\section{PRESENTACIÓN}

Este trabajo es el resultado de los debates y el trabajo final del curso "Políticas de articulación, docencia, investigación, extensión, y transferencia", seminario desarrollado por el equipo del Consejo Social de la UNLP en el marco de la Especialización en Docencia Universitaria (UNLP). El mismo, intenta recuperar algunas reflexiones colectivas sobre la articulación entre la docencia, la investigación, la extensión y la transferencia que surgieron a partir de nuestro proceso de participación en dicho seminario.

La enseñanza -en tanto transmisión del saber- y la investigación -como productora del saber- permanecen bastante estables en el tiempo; sin embargo, la conceptualización y el significado de la extensión ha mutado a lo largo de los años, especialmente durante la segunda mitad del siglo pasado y la primera década del siglo XXI. Con la reforma del Estatuto del año 2008, se retoma el concepto de extensión como una de las funciones básicas y la Universidad la define como "... un proceso educativo no formal de doble vía, planificada de acuerdo a intereses y necesidades de la sociedad, la toma de decisiones y la formación de opinión, con el objeto de generar conocimiento a través de un proceso de integración con el medio y contribuir al desarrollo social". (UNLP, 2008:9)

En los últimos tiempos, observamos un importante cambio en el sentido y la conceptualización de la extensión. Al respecto, Adriani señala sobre" el giro de la extensión: de la asistencia a la comunidad a la construcción conjunta, de la relevancia del conocimiento académico a la centralidad de los problemas sociales, de la direccionalidad universidad-comunidad a la "doble vía" (Adriani, 2015:232).

“ el giro de la extensión: de la asistencia a la comunidad a la construcción conjunta, de la relevancia del conocimiento académico a la centralidad de los problemas sociales, de la direccionalidad universidad-comunidad a la "doble vía"

Es en este contexto, en el cual se generaron nuevos espacios de diálogo con la comunidad-como los Centros Comunitarios de Extensión 
Universitaria (CCEU), la Escuela Universitaria de Oficios y el Consejo Social- con el objeto de aprovechar el aporte y la mirada de los diferentes sectores y actores sociales en el diseño de las propuestas.

A pesar de que este cambio conceptual fue acompañado por un importante crecimiento cuantitativo y cualitativo de la extensión (la cual comenzó a ocupar y generar nuevos espacios institucionales en la gestión universitaria de la UNLP), nos permitimos problematizar y reflexionar sobre algunas posibles relaciones y vinculaciones entre la docencia, la investigación, la transferencia y la extensión en la Universidad Nacional de La Plata, las cuales serán desarrolladas y debatidas a continuación.

\section{REFLEXIONES Y PROBLEMATIZACIONES EN CUANTO A LA ARTICU- LACIÓN EXTENSIÓN-INVESTIGACIÓN- DOCENCIA.}

Entendiendo como problematización, no sólo marcar obstáculos o plantear problemas, sino también crear herramientas, estrategias y/o acciones para resolverlos, retomaremos algunas de las reflexiones para problematizar sobre lo que consideraríamos como algunas posibles limitaciones encontradas en cuanto a la articulación entre las funciones de la Universidad. Presentaremos a continuación algunas referencias en cuanto a la integralidad y la necesidad de vincular extensión/formación de grado/investigación, la formación profesional descontextualizada y la falta de un reconocimiento real de las prácticas de extensión.

Teniendo en cuenta los cambios en la producción de conocimiento y su vinculación con las funciones universitarias, Judith Sutz (2013) refiere a la integralidad "como un espacio de preguntas recíprocas que implica preguntas que la enseñanza le hace a la investigación, que la investigación le realiza a la extensión, y que la extensión le realiza a cualquiera de las otras dos funciones. Asimismo, todas las funciones se formulan preguntas a sí mismas y a todas las demás" (P43)

\section{integralidad "como un espacio de preguntas recíprocas que implica preguntas que la enseñanza le hace a la investigación, que la investigación le realiza a la extensión, y que la extensión le realiza a cualquiera de las otras dos funciones. Asimismo, todas las funciones se formulan preguntas a sí mismas y a todas las demás"}

En primer lugar, concluimos en que una gran limitación para lograr la integralidad -a la que refería Sutz- se podría encontrar en la desinformación y el desconocimiento general por parte de las y los docentes universitarios respecto a cómo se estructura y gestiona la 
extensión universitaria. En ese sentido, en los encuentros del seminario, la mayoría de las y los cursantes (de diversas unidades académicas) coincidimos en el desconocimiento de la existencia y la función tanto del Consejo Social, como de los CCEU. En nuestro caso, además desconocíamos la existencia y las formas de convocatorias ordinarias y extraordinarias para la presentación de proyectos de extensión.

Entendemos que este obstáculo podría ser abordado de manera simple, trabajando en el área comunicacional desde la Universidad y hacia las distintas unidades académicas, por ejemplo, unificando la información en las páginas web de las facultades. Al mismo tiempo, retomando la idea de integralidad, se podrían articular actividades que convoquen a docentes, investigadores y extensionistas (teniendo en cuenta que una persona podrá ejercer más de un rol), para compartir experiencias, avances, puntos de vistas, dificultades e incluso proyectos conjuntos.

En relación a las propuestas de mejora de las estrategias de comunicación, consideramos que por su trabajo territorial los centros de estudiantes tienen un gran caudal de información acerca de las necesidades de la comunidad y, al mismo tiempo, una gran llegada a las y los alumnos. Pero no sucede así con las y los docentes que podrían impulsar proyectos que aborden esas necesidades. Una forma de subsanar este obstáculo comunicacional podría ser la implementación de una base de datos creada por los centros de estudiantes, donde se vuelque la información y que sea de libre acceso a todos los interesados, así los diferentes grupos de docentes podrían acceder y presentar sus propuestas.

En referencia a la integralidad y la complejidad de la realidad social Romano (2013) sugiere que... "si bien se habla de integralidad, cuando se observa la forma del relacionamiento institucional, se ve cómo se procesa la división del trabajo entre las diferentes instituciones públicas y parece que se siguen reproduciendo dinámicas inerciales". (P 102). Frente a esta problemática, es posible plantearse ¿Cómo generar espacios de vinculación entre distintas disciplinas para construir y abordar la complejidad y la diversidad de situaciones que la realidad social demanda? Pensamos que el primer paso debe ser el de construir el problema desde distintos campos interdisciplinares para dar respuestas.

La realidad social es demasiado compleja para abordarla desde una única disciplina. Requiere de articulación de saberes, experiencias y miradas. A su vez, es importante pensar qué sectores podrían componer el trabajo interdisciplinario/interactoral. Existen diversos proyectos e iniciativas construidas por otras instituciones (organizaciones ajenas a la Universidad) que pueden aportar a la agenda comunitaria. Si bien no requieren necesariamente de la Universidad, podrían enriquecerse con los aportes, para articular los saberes académicos con las problemáticas sociales, con el territorio y la comunidad y viceversa. 
El segundo problema en el que coincidimos es que un gran obstáculo para el fortalecimiento del vínculo extensión - formación de grado - investigación continúa siendo la falta de reconocimiento de la actividad extensionista. Más allá de que en los últimos años fue revalorizada y jerarquizada la extensión universitaria en los distintos ámbitos de la Universidad, esta falta de reconocimiento se traduce por un lado en el desconocimiento por parte de las y los estudiantes (los cuales en su mayoría desconocen de la extensión como parte de la formación) y de los propios docentes y las cátedras para buscar una posible articulación con la investigación y la formación.

Lo mismo ocurre para las y los docentes-investigadores, dado que las actividades de extensión no poseen el mismo peso y valor que tienen las actividades de investigación y formación, generando pues que la prioridad sean los proyectos de investigación más que los de extensión. Eso se observa en que estos no son considerados de relevancia, o por lo menos no tienen el mismo valor, al momento de evaluar la actividad profesional de un concurso docente'.

Por esta razón, creemos que la implementación de instrumentos que permitan valorar la actividad de extensión sería fundamental para promover su crecimiento. Según nuestro conocimiento, son escasos -o nulos- los cargos docentes con extensión de la dedicación para la realización de tareas de extensión. La creación y otorgamiento de estos cargos también sería crucial para fomentar el desarrollo de la extensión universitaria y su articulación con la docencia y la investigación; lo mismo sucedería con los aportes de los Programas de Incentivos a Docentes Investigadores, no existiendo dichos incentivos para la extensión. Si la extensión universitaria fuese considerada como una actividad de mayor relevancia en el quehacer de las y los docentes-investigadores, habría mucho más interés por parte de los mismos en presentar proyectos de incumbencia en el área extensionista.

Otra problemática que surge es la formación profesional descontextualizada, una formación meramente académica que resulta "incompleta" para dar respuesta a la complejidad de las demandas sociales y a la elaboración de políticas públicas, programas y actividades. Al respecto, Camilloni afirma que “... en una época en la que la simple actualización de

1 En el caso de la Facultad de Medicina, el reglamento de concursos docentes para proveer cargos de profesor ordinarios no menciona en ninguno de sus artículos la palabra extensión. Si bien algunas facultades utilizan grillas para el otorgamiento de puntajes en los concursos docentes e incluyen a la extensión como actividad a ser valorada, en la Facultad de Ciencias Médicas aún no está reglamentada dicha modalidad. A diferencia de la mencionada Facultad, en el caso de la Facultad de Humanidades y Ciencias de la Educación sí existen ítems específicos relacionados a considerar la extensión; sin embargo, en la mayoría de los casos queda relegada como criterio a ser valorado, por debajo de la docencia y la investigación. 
los currículos en términos de necesidades científicas, tecnológicas y profesionales presentes es insuficiente". (Camilloni, 2013:11).

A pesar de que en los últimos años se ha dado lugar al reconocimiento de la importancia del trabajo interdisciplinario para favorecer la construcción de respuestas dirigidas a la sociedad en general, entendemos que aún existe una escasa incorporación de dichas actividades de formación, contenidos curriculares y asignaturas de carácter obligatorio, destinadas a la trasmisión de metodologías y experiencias en extensión universitaria.

Cano Menoni (2014) describe" ...el compromiso social de la universidad se realiza a través del conjunto de su quehacer educativo, científico y cultural, y se expresa en sus políticas de acceso, su oferta académica, sus agendas de investigación, modelos educativos y prioridades científicas. Pero por su naturaleza, la función de extensión universitaria se vincula de un modo más directo con la idea del compromiso social de las universidades" (P 12).

\section{...el compromiso social de la universidad se realiza a través del conjunto de su quehacer educativo, científico y cultural, y se expresa en sus políticas de acceso, su oferta académica, sus agendas de investigación, modelos educativos y priorida- des científicas. Pero por su naturaleza, la función de extensión universitaria se vincula de un modo más directo con la idea del compromiso social de las universidades"}

Colectivamente hemos debatido sobre nuestras experiencias por cada unidad académica (como estudiantes y como profesores) y concluimos que, en nuestra formación de grado, en ningún caso accedimos -ni acceden los y las estudiantes de hoy- a espacios y contenidos curriculares (sean asignaturas, seminarios, etc.) dentro de los planes de estudios de cada carrera relacionados al desarrollo de la extensión².

Esta situación demuestra que a pesar de que la Universidad considera a la extensión como un pilar de sus funciones, la misma aún no resulta considerada como una actividad perteneciente a la currícula de las carreras. Por eso creemos fundamental avanzar en la integración de las actividades de extensión en la formación de grado (currículos universitarios y planes de estudios) porque entendemos que "La formación debe ser muy actualizada para abrir las puertas a la pro-

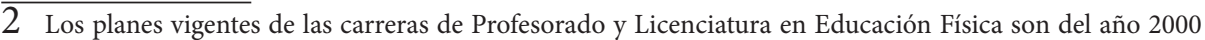
-momento en el cual, tal como mencionamos anteriormente, la extensión no estaba desarrollada ni pensada como en la actualidad, por lo cual no fue incluida dentro de los mismos- y si bien desde hace algunos años se viene trabajando sobre la reforma de los planes de estudios de dichas carreras, destacamos que no se ha debatido la inclusión de estos espacios en el nuevo plan para la formación de las y los estudiantes. En el caso de la Licenciatura en Nutrición, el plan de estudios es del año 2012, año en que inició la carrera; y a pesar de ser más actual, tampoco incluye a la extensión.
} 
ducción de conocimientos, preocupada por promover la comprensión de las redes que conforman la sociedad civil y el Estado y por mostrar la conexión del conocimiento académico y profesional con la ciencia, la tecnología y los mundos natural y social, porque el conocimiento universitario ya no se encuentra encerrado en el ámbito de las disciplinas académicas" (Camilloni, 2013:12).

\section{"La formación debe ser muy actualizada para abrir las puertas a la producción de conocimientos, preocupada por promover la comprensión de las redes que conforman la sociedad civil y el Estado y por mostrar la conexión del conocimiento académico y profesional con la ciencia, la tecnología y los mundos natural y social, porque el conocimiento universitario ya no se encuentra encerrado en el ámbito de las disciplinas académicas"}

Además, una formación que esté fuertemente comprometida con una perspectiva integral de derechos humanos y sociales probablemente colabore a la concientización de las y los estudiantes y docentes respecto a cómo desde la Universidad se pueden promover y desarrollar prácticas orientadas a fortalecer el lazo con la comunidad aportando a las necesidades de la misma, apoyando el compromiso social de la Universidad.

Compromiso, para el cual resulta indispensable resignificar y atender la relevancia que las actividades y proyectos de extensión adquieren en los procesos de formación universitaria. Asimismo sobre la participación de los y las estudiantes en las actividades de extensión, Romano señala "...es importante considerar que el conocimiento se puede poner en juego en un espacio de aprendizaje más allá del aula y más allá de la práctica profesional para innovar en los formatos de enseñanzas más integrales, teniendo en cuenta la producción de conocimientos con otros, de ese saber que no se tiene." (Romano, 2013:85).

Ante una realidad social diversa y compleja, se vuelve necesario la articulación de las diferentes Secretarías de Extensión de las distintas unidades académicas para la ejecución de proyectos requeridos por la agenda comunitaria con el objetivo de poner en contacto a las diferentes áreas participantes de los proyectos, permitiendo así un abordaje interdisciplinario que logre optimizar recursos y potenciar acciones.

Otro aspecto que atenta contra la integralidad de las prácticas es el uso no democrático de los espacios físicos de las facultades. Es muy común observar la existencia de espacios "destinados a la investigación" donde las y los estudiantes no pueden acceder y espacios exclusivos para el ejercicio de la docencia. En un contexto en el que se empieza a limitar la posibilidad de crecimiento de los grupos de investigación 
por problemas de espacio, parece impensado poder articular la participación de las y los estudiantes en los proyectos de investigación o llevar a cabo proyectos de extensión en el ámbito de los espacios físicos de la facultad. Una forma de resolver este obstáculo sería disminuir los espacios de usos "exclusivos" para una única actividad, por ejemplo, para docencia; además, se podría contar con un cronograma de los espacios al que tengan acceso las áreas de docencia, investigación y extensión, para optimizar la utilización. Es de gran relevancia considerar la infraestructura edilicia para las actividades que la Universidad propone, dado que resulta un impedimento para el desarrollo de los proyectos la falta de espacios donde ejecutarlos.

Planteamos que sería útil la creación de un "banco de tecnologías, desarrollos y habilidades", donde las y los docentes e investigadores interesados en participar en proyectos de extensión, puedan volcar propuestas sobre actividades que podrían nutrir estos proyectos. La existencia de este tipo de bancos de experticia y de estructuras complejas como los centros comunitarios y su agenda, propiciaría el diálogo entre los actores que plantean el problema y aquellos que cuentan con herramientas para solucionarlos. De igual manera, este tipo de banco podría favorecer el desarrollo de la interdisciplina.

Otra reflexión importante que se generó fue sobre nuestras prácticas de enseñanza dentro de la Universidad. Entendemos a la formación y a la educación, no como una acumulación de conocimientos, sino como una construcción social del conocimiento para "la formación de profesionales críticos, reflexivos y socialmente responsables" (Rafaghelli, 2013:23), diferenciándose de la enseñanza en términos de donación, acumulación o imposición, perspectiva por la cual niega a quien aprende como sujeto social con capacidad para crear y transformar, reflexionar y actuar. En esa construcción social del conocimiento, consideramos indispensable la articulación de la formación y la investigación con las actividades de extensión, ya que "...la extensión universitaria puede aportar ... profundizando el sentido de lo público universitario y democratizando la propia contienda por el sentido de la transformación de la universidad." (Cano Menoni, 2014:53), sobre todo sabiendo del fuerte compromiso de la Universidad con la formación de agentes de transformación social, para abordar dicha realidad social.

consideramos indispensable la articulación de la formación y la investigación con las actividades de extensión, ya que "...la extensión universitaria puede aportar ... profundizando el sentido de lo público universitario y democratizando la propia contienda por el sentido de la transformación de la universidad." 


\section{CONCLUSIÓN}

Repensar la función social de la Universidad Pública requiere defender los valores propios de la educación pública. En el actual contexto sociocultural, la Universidad es percibida como un óptimo espacio de formación, no sólo de carácter profesional y cultural -en su sentido más amplio- sino también de carácter humano, y, por ende, ético y moral. Una Universidad de calidad y de servicio público es aquella que hace más digna la sociedad, convirtiendo a sus estudiantes tanto en excelentes profesionales, como en ciudadanos y ciudadanas cada vez más cultos y críticos. (Gauna, 2010)

\section{Repensar la función social de la Universidad Pública requiere defender los valores propios de la educación pública.}

Como mencionan Andrini y Liaudat (2019), es necesario discutir políticamente, con soberanía, las decisiones científicas y tecnológicas que se toman a todo nivel. Politizar la ciencia y la tecnología. Los autores plantean la necesidad de discutir el para qué y para quiénes se producen conocimientos, tener un pensamiento/ hacer situado que traspase los muros de una investigación científica y tecnológica globalizada para dar lugar a la construcción de una agenda local y regional circunscripta por un proyecto nacional orientador y conforme a un modelo deseable de sociedad. Con todas las dimensiones que la abarcan, esto es una tarea fundamentalmente política.

En ese sentido, consideramos coincidiendo con Cano Menoni (2014) en que implican un desafío "los procesos de transformación de la universidad y la resignificación de su compromiso social de modos alternativos al discurso hegemónico capitalista de la modernidad conservadora, así como la racionalidad productivista de algunos de los discursos desarrollistas o neo -desarrollistas." (Cano Menoni,2014:58).

Esto requiere abordar no sólo los aspectos pedagógicos sino también los planos político, metodológico y epistemológicos, procurando profundizar su vinculación con los procesos de enseñanza y creación de conocimiento y consolidar vínculos de trabajo de largo plazo con organizaciones sociales y la comunidad, para lo cual la articulación entre la docencia, investigación, extensión y transferencia se vuelve vital.

Por esto, insistimos en que resulta necesario generar saber y conocimiento a partir de la vinculación de la extensión, la investigación y la docencia. Las tres funciones de la Universidad, estrechamente vinculadas, pero no reconocidas todavía de igual modo. 
insistimos en que resulta necesario generar saber y conocimiento a partir de la vinculación de la extensión, la investigación y la docencia. Las tres funciones de la Universidad, estrechamente vinculadas, pero no reconocidas todavía de igual modo.

Sobre esta disparidad resta reflexionar pero ya plantear la necesidad de equiparar las funciones de la Universidad visibiliza el problema. 


\section{BIBLIOGRAFIA}

Adriani, L, en Carballo, C. (2015) “Diccionario Crítico de la Educación Física Académica." Entrada Extensión. Editorial Prometeo, Buenos Aires.

Andrini L, Liaudat S (2019) ¿Por qué discutir políticamente la ciencia y la tecnología? En M. Speroni (coord.) “Política: apareces en el aire, dimensión misteriosa y escurridiza de la experiencia humana" En _la_apropiacion_del_conocimiento_desde_la_universidad

Camilloni, A. (2013). La Inclusión de la educación experiencial en el curriculum universitario. https://bibliotecavirtual.unl.edu.ar:8443/bitstream/handle/11185/1223/extension_interior_pag_11_21.pdf?sequence=2\&isAllowed=y

Cano Menoni, JA (2014). La extensión universitaria en la transformación de la universidad latinoamericana del siglo XXI: disputas y desafíos. CLACSO, Buenos Aires.http://biblioteca.clacso.edu.ar/clacso/becas/20141202093928/ensayo_ cano_premio_pedro_krotsch.pdf

Codner, D; Becerra, P; Díaz, A. (2012). La transferencia tecnológica ciega: desafíos para la apropiación del conocimiento desde la Universidad. Redes, vol.18 nro 35 pp 161-171, Bernal. https://www.researchgate.net/publication/266142123_ La_transferencia_tecnologica_ciega_desafios_para

Entredichos. Intervenciones y Debates en Trabajo Social. Facultad de Trabajo Social, UNLP, Dossier N 6, 2019. http://entredichos.trabajosocial.unlp.edu.ar/ wp-content/uploads/sites/6/2019/09/Articulo-3.pdf

Gaúna, E. A (2010). Universidad y Construcción de Ciudadanía: 'Perspectiva sobre la política, lo político, la participación y el ejercicio de la ciudadanía de los estudiantes de la UNNE.' VI Jornadas de Sociología de la UNLP. Universidad Nacional de La Plata. Facultad de Humanidades y Ciencias de la Educación. Departamento de Sociología, La Plata. http://www.memoria.fahce.unlp.edu. ar/trab_eventos/ev.5572/ev.5572.pdf

Rafaghelli M. (2013). La dimensión pedagógica de la extensión. Integración docencia y extensión. Otra forma de enseñar y de aprender. 1a ed. Santa Fe: Universidad Nacional del Litoral. Pág. 22-37 http://biblioteca.clacso.edu.ar/Argentina/fcjs-unl/20171101043348/pdf_1172.pdf

Romano, A. (2013) A. Sobre los espacios de formación integral en la Universidad. Una perspectiva pedagógica. Integralidad: tensiones y perspectivas. Cuadernos de Extensión № 1. Pág. 85-107. https://www.extension.udelar.edu.uy/ wp-content/uploads/2017/11/Cuaderno-n\%C2\%B01-integralidad.pdf 
de preguntas recíprocas. Integralidad: tensiones y perspectivas. Cuadernos de Extensión № 1. Pág. 43-60. https://www.extension.udelar.edu.uy/wp-content/ uploads/2017/11/Cuaderno-n\%C2\%B01-integralidad.pdf

\section{$\mathrm{CV}$}

*Profesor en Educación Física (FaHCE-UNLP), Estudiante Especialización en Docencia Universitaria (EDU) y Maestrando en Deporte (FaHCE-UNLP). Profesor Titular de Educación Física Colegio Liceo Víctor Mercante (UNLP) y Ayudante Diplomado interino Teoría de la Educación Física II. (FaHCE-UNLP). Participante en diversos Proyectos de Extensión: e Integrante diversos Proyectos de Investigación acreditados en la Facultad de Humanidades y Ciencias de la Educación (UNLP).

**Biotecnóloga y Dra de la Facultad de Ciencias Exactas (UNLP). Docente-Investigador UNLP. Jefe de trabajos prácticos con dedicación exclusiva de Bioquímica y Biología Molecular en la carrera de Medicina, Facultad de Ciencias Médicas (UNLP), Jefe de trabajos prácticos de Bioquímica, Licenciatura en Nutrición Facultad de Ciencias Médicas (UNLP). Docente en cursos de posgrado y maestrías en la Facultad de Ciencias Médicas (UNLP). Integrante de Proyectos de Investigación acreditados en la Facultad de Ciencias Médicas (UNLP) Estudiante de la Especialización en Docencia Universitaria, UNLP.

***icenciada en Nutrición (Universidad de Buenos Aires) Profesora Adjunta de Técnica Dietoterápica en la Licenciatura en Nutrición, Facultad de Ciencias Médicas (UNLP), Participante en Proyecto de Extensión de la Facultad de Ciencias Médicas (UNLP), Docente a cargo del voluntariado de la Licenciatura en Nutrición (FCM-UNLP), Estudiante de la Especialización en Docencia Universitaria, UNLP.

****Bioquímica y Dra. de la Facultad de Ciencias Exactas (UNLP), Investigadora Adjunta CONICET, Profesora Adjunta de Bioquímica en la Licenciatura en Nutrición, Facultad de Ciencias Médicas (UNLP), Profesora Adjunta de Introducción a la Nutrigenómica, Licenciatura en Nutrición (UNLP), Docente en cursos de posgrado y maestrías en la Facultad de Ciencias Médicas (UNLP). Estudiante de la Especialización en Docencia Universitaria, UNLP. 